\title{
GLOBAL EXISTENCE OF SOLUTIONS OF THE YANG-MILLS EQUATIONS ON GLOBALLY HYPERBOLIC FOUR DIMENSIONAL LORENTZIAN MANIFOLDS*
}

\author{
PIOTR T. CHRUŚCIEL ${ }^{\dagger}$ AND JALAL SHATAH ${ }^{\ddagger}$
}

\begin{abstract}
We prove global solvability of the Cauchy problem for the Yang-Mills equations on smooth globally hyperbolic four dimensional Lorentzian manifolds.
\end{abstract}

1. Introduction. In a classical paper [9], Eardley and Moncrief have shown that solutions of the Yang-Mills equations on Minkowski space-time (with a compact gauge group) do not develop singularities in finite time, provided the initial data are sufficiently regular. This result has been generalized ${ }^{1}$ to the Einstein cylinder $\mathbb{R} \times \mathbb{S}^{3}$ in $[6,8,4]$, and to anti-de-Sitter space-time in [1], making use of the conformal invariance of the Yang-Mills equations. It is of interest to enquire whether or not this result remains true for general globally hyperbolic Lorentzian manifolds; such a result in dimensions $1+1$ and $2+1$ has been established, for quite a large class of manifolds, via direct energy methods, in [3]. It is the purpose of this paper to show that global existence holds true in $3+1$ dimensions: we show global solvability of the Cauchy problem for the Yang-Mills equations on any globally hyperbolic Lorentzian manifold ${ }^{2}(M, g)$.

It is convenient to start with a description of the geometric context. Recall that a Lorentzian manifold is globally hyperbolic if it admits a Cauchy surface $\Sigma$, i.e. a hypersurface $\Sigma \subset M$ that is intersected precisely once by every inextendible causal curve. For such manifolds there exists a smooth time function $t$ on $M$ such that $\Sigma=\{t=0\}$, with each level set $\Sigma_{t}$ of $t$ being a Cauchy surface [11, 16]. Moreover, flowing along the gradient of $t$ one obtains a diffeomorphism between $M$ and $\mathbb{R} \times \Sigma$.

Let $G$ be any connected Lie group with Lie algebra $\mathfrak{g}$. Throughout we shall assume that 1$) \mathfrak{g}$ is compact, that is, $\mathfrak{g}$ admits an Ad-invariant positive scalar product $k(\cdot, \cdot)$ (that will be the case if, e.g., $G$ is compact). 2) $\mathfrak{g}$ has a faithful representation as a subalgebra of the algebra of matrices over some finite dimensional vector space $V$, so that the bracket operation corresponds to the commutator of matrices, with the adjoint representation of $G$ acting on $\mathfrak{g}$ as an appropriate product of matrices. Let $P$ be a $G$-principal bundle over $M$ and let $P^{\Sigma}$ be the pull-back of $P$ to a Cauchy surface $\Sigma$ Choose any smooth connection on $P$, let $X$ denote the horizontal lift of the gradient of $t$ to $P$, we can identify $P$ with $\mathbb{R} \times P^{\Sigma}$ by flowing along the integral curves of $X$. This leads to the commutative diagram:

\footnotetext{
${ }^{*}$ Received April 16, 1997; accepted for publication (in revised form) October 6, 1997.

†Département de Mathématiques, Faculté des Sciences, Parc de Grandmont, F37200 Tours, France (Chrusciel@Univ-Tours.Fr). Supported in part by a Polish Research Council grant number 2 P301 05007.

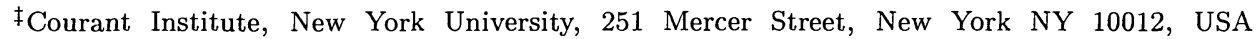
(shatah@cims.nyu.edu). Supported in part by N.S.F. DMS 9401558.

${ }^{1}$ Some further improvements of the Eardley-Moncrief theorem can be found in $[12,14]$.

${ }^{2}$ We use the signature -+++ , and we assume that both the manifold and the metric are smooth. $M$ is moreover assumed to be paracompact, Hausdorff, and time oriented.
} 


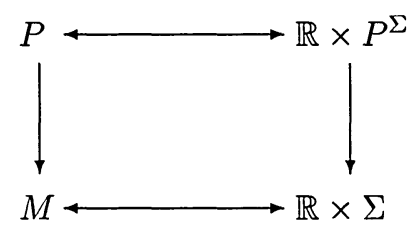

Given a trivialisation of $P$ over a coordinate patch $\mathcal{U}$ of $M$, a connection $A$ on $P$ can be expressed as a $\mathfrak{g}$-valued one form on $\mathcal{U}$,

$$
A=A_{\mu}(x) d x^{\mu}, \quad A_{\mu}(x) \in \mathfrak{g},
$$

and the curvature $F$ as the $\mathfrak{g}$-valued two form given by

$$
F_{\mu \nu}=\partial_{\mu} A_{v}-\partial_{\nu} A_{\mu}+\left[A_{\mu}, A_{\nu}\right]
$$

A connection $A$ is a Yang-Mills connection if it satisfies

$$
\mathcal{D}_{\mu} F^{\mu \nu}=F_{; \mu}^{\mu \nu}+\left[A_{\mu}, F^{\mu \nu}\right]=0 .
$$

Here and everywhere the symbol $\mathcal{D}$ denotes a space-time and gauge covariant derivative, while $\nabla_{\mu}$ or the addition of a sub- or superscript " $\mu^{\prime \prime}$ denotes a space-time covariant derivative.

The Cauchy problem for the Yang-Mills equations on $M$ consists in prescribing on $P^{\Sigma}$ a connection field $A^{\Sigma}$ together with an Ad-equivariant $\mathfrak{g}$-valued one form $E$. (Loosely speaking, $E$ represents the time-derivative of the connection form on $\Sigma$.) Moreover $A^{\Sigma}$ and $E$ are assumed to satisfy the Yang-Mills constraint ${ }^{3}$ equation,

$$
\mathcal{D}_{i} E^{i}=0 \text {. }
$$

We shall say that a connection field is of differentiability class $H_{\mathrm{loc}}^{k}(\Sigma)$ if there exists a covering of $\Sigma$ by coordinate balls $\mathcal{U}_{\alpha}$ together with trivialisations of $\left.P^{\Sigma}\right|_{U_{\alpha}}$ such that in local coordinates the components $A_{i}^{\Sigma}$ of $A^{\Sigma}$ are in $H_{\text {loc }}^{k}\left(\mathcal{U}_{\alpha}\right)$. A similar definition is used for $E$. A connection $A$ on $P$ will be called a Yang-Mills development of $\left(A^{\Sigma}, E\right)$ if $A$ is a solution of the Yang-Mills equations (1.1), such that the pull-back of $A$ to $P^{\Sigma}$ coincides with $A^{\Sigma}$, and such that the pull-back of $n \vee F$ to $P^{\Sigma}$ coincides with $E$. (We also impose some differentiability conditions on $A$, which are made precise in Section 4.) Here $n$ is the horizontal lift to $P$ of the field of future directed unit normals to $\Sigma$, and $\vee$ denotes contraction of a vector with a form. In this paper we prove the following:

THEOREM 1.1. Let $P$ be a principal bundle over a smooth globally hyperbolic four dimensional Lorentzian manifold $(M, g)$ with structure group $G$, whose Lie algebra $\mathfrak{g}$ is compact. Let $\Sigma$ be a Cauchy surface in $M$ and let $P^{\Sigma}$ be the pull-back of $P$ to $\Sigma$. Let $A^{\Sigma} \in H_{\mathrm{loc}}^{k+1}(\Sigma)$ be a connection on $P^{\Sigma}$ and let $E \in H_{\mathrm{loc}}^{k}(\Sigma)$ be an Ad-equivariant $\mathfrak{g}$-valued one form on $P^{\Sigma}, k \geq 2$, satisfying the constraint (1.2). Then there exists a unique Yang-Mills development $A$ of $\left(A^{\Sigma}, E\right)$ on $P$. (If the initial data are smooth then the Yang-Mills development is smooth.)

To establish theorem 1.1 we adapt several of the Eardley-Moncrief ideas to the curved space-time setting. Let us highlight some elements of our proof:

\footnotetext{
$[4,2]$.

${ }^{3}$ Some results concerning existence of solutions of the constraint equation (1.2) can be found in
} 
- One of the key ingredients of the Eardley-Moncrief proof is the use of the Cronström gauge. We also use this gauge, and this carries over to curved space-time without introducing any difficulties.

- Eardley and Moncrief's strategy is to establish an $L^{\infty}$ a priori estimate on the Yang-Mills curvature field $F$, using the spherical means representation of solutions of the wave equation. We follow a similar strategy, replacing the spherical means integral by a representation formula of Friedlander [10]. There are two differences between those formulae and the Minkowski spacetime ones: First, there are more terms to deal with, because of a solid-cone integral in a generic curved space-time which is absent in Minkowski spacetime. This introduces some tediousness to the proof but does not lead to any difficulties because the solid-cone integral terms that arise can be estimated in a rather straightforward way. Next, the formulae of Friedlander are valid only in causal domains (as defined in [10]), while the spherical means are valid in the whole of Minkowski space-time. To handle that issue, roughly speaking we first obtain an $\|F\|_{H_{\text {loc }}^{2}}$ a priori estimate in causal domains, and use a globalisation argument essentially due to Choquet-Bruhat and Geroch [5] to obtain global existence on any globally hyperbolic manifold. This is here that the condition of global hyperbolicity of the space-time enters in our argument.

- It turns out that the arguments used by Eardley-Moncrief to control all the terms that arise in the light cone integral carry over to the curved space-time case, except for the term $\left[A_{; \alpha}^{\alpha}, F_{\mu \nu}\right]$. To take care of that term we have to use a completely different argument, requiring simultaneous control of $\|F\|_{L_{\text {loc }}^{\infty}}$ and of a $H_{\text {loc }}^{2}$ semi-norm of $F$. The observation that this term can be handled in this way is the main new idea of this paper.

- We note, finally, that a slight difficulty is introduced by the constraint part of the Yang-Mills equations. While there are several ways to handle this problem, we use here the observation of Kapitanskii and Goganov [12] that one can obtain Eardley-Moncrief type estimates for appropriately modified Yang-Mills equations, in which the constraint part of the equations is only partially satisfied.

This paper is organized as follows: In Section 2 we review Friedlander's representation formulae. In Section 3 we derive various integral a priori estimates. including the mixed $\|F\|_{L_{\text {loc }}^{\infty}}$ and $H_{\text {loc }}^{2}$ estimates. The proof of Theorem 1.1 is given in Section 4 .

Notations. Given a time oriented four dimensional Lorentzian manifold $(M, g)$, we denote local coordinates by $x=\left(x^{0}, x^{1}, x^{2}, x^{3}\right)=\left(x^{0}, \mathrm{x}\right), r=|\mathrm{x}|$, and the volume element by $\mu=\sqrt{|g|} d x$. Given a hypersurface $S=\{x ; f(x)=0\}, \mu_{f}$ will denote the Leray form associated with $S$, i.e. $\mu=d f \wedge \mu_{f}$. We use the following notations for functions and tensors:

$X(f)=X^{\alpha} \partial_{\alpha} f$ the derivative of $f$ in the direction of a vector $X$, $\nabla f=\left\{g^{\mu \nu} \partial_{\nu} f\right\}$ the gradient of a function $f$, $\nabla_{\alpha} T_{s}^{r}=T_{s ; \alpha}^{r}$ the covariant derivative of a tensor field $T$ of type $\{r, s\}$, $\nabla_{X} T_{s}^{r}=X^{\alpha} T_{s ; \alpha}^{r}$ the derivative of $T$ in the direction of a vector $X$, $\langle T, S\rangle=$ the inner product of $T$ and $S$ with respect to the metric $g$.

We will assume that $M$ is globally hyperbolic, which implies that $M$ is foliated by space-like hypersurfaces $\Sigma_{t}$, which are the level surfaces of a smooth time function $t$, 
and denote by $\underline{t}=\frac{d t}{\sqrt{-\langle d t, d t\rangle}}$. On $T M$, the tangent space of $M$, we introduce basis vectors $\left\{\hat{t}, \hat{z}_{1}, \hat{z}_{2}, \hat{z}_{3}\right\}$ where $\hat{t}$ is a unit vector in the direction of $-\nabla t$, and

$$
\langle\hat{t}, \hat{t}\rangle=-1, \quad\left\langle\hat{t}, \hat{z}_{i}\right\rangle=0, \quad\left\langle\hat{z}_{i}, \hat{z}_{j}\right\rangle=\delta_{i j} .
$$

For any vector field $X$, let

$$
|X|^{2}=|\langle\hat{t}, X\rangle|^{2}+\sum\left|\left\langle\hat{z}_{i}, X\right\rangle\right|^{2}
$$

and for a tensor $T,|T|$ is defined in a similar fashion.

Acknowledgements: The first author acknowledges an enlightening collaboration with J. Baez during an early attempt to prove Theorem 1.1.

2. The wave equation on $M$. In this section we will state some basic results about the wave equation on a four dimensional Lorentzian manifold $(M, g)$. We review the explicit formula for the fundamental solution of the wave equation on $M$ of [10]. All of our statements and results are restricted to a geodesically convex neighbourhood $\Omega \subset M$. The derivation of the fundamental solution formula can be found in Friedlander [10].

On a geodesically convex neighbourhood $\Omega$ we denote by $\gamma(p, q)=$ square of the geodesic distance between $p, q \in \Omega$,

$C^{-}(p)=$ past null semicone with vertex $p \in \Omega$,

$J^{-}(p)=$ the closure of the causal past of $p$,

$D\left(t_{0}\right)=\left\{q \in J^{-}(p) ; \quad t(q)=t_{0}<t(p)\right\}$ a cross section of $J^{-}(p)$,

$K\left(t_{1}, t_{2}\right)=\left\{q \in J^{-}(p) ; \quad t_{1} \leq t(q) \leq t_{2}<t(p)\right\}$ a truncated cone,

$M\left(t_{1}, t_{2}\right)=\left\{q \in C^{-}(p) ; \quad t_{1} \leq t(q) \leq t_{2}<t(p)\right\}$ the mantle of the truncated cone $K\left(t_{1}, t_{2}\right)$,

with similar definitions for $C^{+}(p), J^{+}(p)$.

DEFINITION 2.1. Given a point $p \in M$ define null basis vectors on the tangent space $T_{p} M$, as basis vectors $\left\{\hat{\ell}, \hat{m}, \hat{e}_{1}, \hat{e}_{2}\right\}$, that satisfy

$$
\begin{aligned}
& \langle\hat{\ell}, \hat{\ell}\rangle=\langle\hat{m}, \hat{m}\rangle=\left\langle\hat{\ell}, \hat{e}_{i}\right\rangle=\left\langle\hat{m}, \hat{e}_{i}\right\rangle=0 \\
& \langle\hat{\ell}, \hat{m}\rangle=2 \quad \text { and }\left\langle\hat{e}_{i}, \hat{e}_{j}\right\rangle=\delta_{i j} .
\end{aligned}
$$

The forms dual to $\left\{\hat{\ell}, \hat{m}, \hat{e}_{1}, \hat{e}_{2}\right\}$ are denoted by $\left\{\underline{\ell}, \underline{m}, \underline{e_{1}}, \underline{e_{2}}\right\}$. We will always work with the following null basis on $C^{-}(p) \backslash p$. For any $q \in C^{-}(p) \backslash p$, let $\hat{n}$ denote the unit vector orthogonal to $\hat{t}$ such that

$$
\nabla \gamma=a \hat{t}+b \hat{n}
$$

Here the gradient of $\gamma$ is taken with respect to the $q$ variable, $p$ being held fixed. Define $\hat{\ell}=\hat{t}-\hat{n}$, and $\hat{m}=-(\hat{t}+\hat{n})$; then for any orthonormal basis $\left\{\hat{n}, \hat{e}_{1}, \hat{e}_{2}\right\}$ of the tangent space $T_{q} \Sigma_{t(q)}$, we obtain a null basis $\left\{\hat{\ell}, \hat{m}, \hat{e}_{1}, \hat{e}_{2}\right\}$.

The representation formula. The fundamental solution of the wave equation $G^{-}(p, q)$

$$
\square G^{-}:=-\nabla^{\alpha} \partial_{\alpha} G^{-}=\delta(p),
$$

whose support is contained in $J^{-}(p)$, can be computed inside a causal domain $\Omega_{0}$ :

$$
G^{-}(p, q)=U(p, q) \delta_{-}(\gamma)+V^{-}(p, q)
$$


where, in a coordinate chart $(\pi(p)=x$ and $\pi(q)=y)$,

$$
\begin{aligned}
U(x, y) & =\frac{\left|\operatorname{det} \gamma_{x^{i} y^{j}}\right|^{\frac{1}{2}}}{8 \pi|\operatorname{det}(g(x)) \operatorname{det}(g(y))|^{\frac{1}{4}}}, \\
\delta_{-}(\gamma) & =\lim _{\epsilon \rightarrow 0} \delta_{-}(\gamma-\epsilon) .
\end{aligned}
$$

Here $\delta_{-}(\gamma-\epsilon)$ is the Dirac measure supported on the lower branch of the hyperboloid $\gamma=\epsilon$, and, following [10, p. 146], we say that a connected open set $\Omega$ is a causal domain when it is globally hyperbolic and contained in a geodesically convex set. $V^{-}$ is a solution to the characteristic problem

$$
\begin{aligned}
& \square V^{-}=0 \quad \text { in } \quad J^{-}(p), \\
& \left.V^{-}\right|_{C^{-}(p)}=\left.V_{0}\right|_{C^{-}(p)}:=-\left.\frac{1}{4} U(x, y) \int_{0}^{1} \frac{\square U}{U} d s\right|_{C^{-}(p)},
\end{aligned}
$$

where the integral is over a geodesic $c(s)$ joining $p=c(0)$ to $q=c(1)$. In a small causal domain $\Omega_{0}$ the above problem has a solution $V^{-}(p, q) \operatorname{such}$ that $\operatorname{supp} V^{-} \subset$ $\Delta^{-}, V^{-} \in C^{\infty}\left(\Delta^{-}\right)$, where

$$
\Delta^{-}=\left\{(p, q): \quad q \in J^{-}(p)\right\}
$$

is the closure of one component of $\{(p, q) ; \gamma(p, q)>0\}$. The function $q \rightarrow V^{-}(p, q)$ is supported in $J^{-}(p)$ and is $C^{\infty}$ at the boundary of its support.

Using this fundamental solution we can find a representation for solutions of the Cauchy problem,

$$
\begin{gathered}
\square u=f, \\
\left.u\right|_{D}=u_{0}, \\
\left.\nabla u\right|_{D}=u_{1},
\end{gathered}
$$

where $D \subset \Sigma_{a} \cap \Omega_{0}$ is a space-like hypersurface, and $X\left(u_{0}\right)=\left\langle X, u_{1}\right\rangle$ for any vector field $X$ tangent to $D$.

For $p \in \Omega_{0}$ let $K(p)$ be the cone of vertex $p$ and whose base lies on $D$, that is, $K(p)=J^{-}(p) \cap J^{+}(D)$, where $J^{+}(D)$ denotes the set of points which lie to the causal future of $D$. Let $C(p)=C^{-}(p) \cap K(p)$ be the mantle of this cone, and let $S=\Sigma_{a} \cap C^{-}(p)$ be the topological boundary of $C(p)$. Then $u$ is given by

$$
u(p)=u_{h}(p)+\int_{C(p)} U(p, q) f(q) \mu_{\gamma}+\int_{K(p)} V^{-}(p, q) f(q) \mu,
$$

where $\mu_{\gamma}=$ the Leray form associated with $C(p)$, i.e., $\mu=d \gamma \wedge \mu_{\gamma}$, and $u_{h}(p)$ is the solution of the homogenous wave equation with the same initial data. To express $u_{h}(p)$ explicitly in terms of the initial data we need to define the following. Let $C^{+}(S)$ be the null hypersurface whose normal on $S$ is given by $\hat{m}$, and $S_{\varepsilon}$ the intersection of $C^{+}(S)$ and the set $\{q ; \gamma(p, q)=\varepsilon\}$. Denote by $\mu_{S}$ and $\mu_{S_{\varepsilon}}$ the volume elements induced by the the metric $g$ on the surface $S$ and $S_{\varepsilon}$ respectively. On $S$ define the functions $\rho$ and $\theta$

$$
\begin{aligned}
& \rho=\langle\hat{m},-\nabla \gamma\rangle / 2, \\
& \theta \mu_{S}=\left.\rho \frac{d}{d \varepsilon} \mu_{S_{\varepsilon}}\right|_{\varepsilon=0},
\end{aligned}
$$


then

$$
\left.u_{h}(p)=\int_{S}\left[\frac{1}{\rho} U[\langle\hat{m}, \nabla u\rangle+\theta u)\right]+V^{-} u\right] d S+\int_{D(a)}\left(V^{-} t^{a} \nabla_{a} u-u t^{a} \nabla_{a} V^{-}\right) \mu_{a} .
$$

If we parametrize the cone $C(p)$ by $(\zeta, \omega) \in \mathbb{R}^{+} \times \mathbb{S}^{2}$, the solution $u$ can be expressed in the following way. Let $\hat{\xi}$ be a unit vector orthogonal to $\hat{t}(p)$, parameterized by $\omega \in \mathbb{S}^{2}$. For any past directed null vector $\hat{v}$ of the form $\hat{v}=-\hat{t}+\hat{\xi}$, and $\zeta \in \mathbb{R}^{+}$ let $q=\exp _{p}(\zeta \hat{v})$. Since we are in a geodesically convex set, the $\exp _{p}$ map is a diffeomorphism from $C(p) \backslash p$ into $\left(0, \zeta_{0}(\omega)\right] \times \mathbb{S}^{2}$, where $\exp _{p}\left(\zeta_{0}(\omega) \hat{v}\right) \in S$. In these coordinates

$$
\begin{aligned}
U \mu_{\gamma} & =\frac{\kappa(\zeta, \omega)}{\zeta} \mu_{\ell}=\tilde{\kappa}(\zeta, \omega) \zeta d \zeta \wedge d \omega \\
\mu_{S} & =\chi\left(\zeta_{0}(\omega), \omega\right) \zeta_{0}(\omega)^{2} d \omega \\
\rho & =\tilde{\kappa}(\zeta, \omega) \zeta \\
\theta & =\frac{\tilde{\theta}(\zeta, \omega)}{\zeta}
\end{aligned}
$$

where $\kappa, \tilde{\kappa}, \chi, \tilde{\chi}$, and $\tilde{\theta}$ are smooth positive functions, and where $\mu_{\ell}$ denotes the Leray form on $C^{-}(p) \backslash p$. Equation (2.3) can be written as

$$
\begin{aligned}
& u(p)=u_{h}(p)+\int_{C(p)} f(\zeta, \omega) \frac{\kappa(\zeta, \omega)}{\zeta} \mu_{\ell}+\int_{K(p)} V^{-} f \mu \\
& u_{h}(p)=\int_{S}\left(\frac{1}{\zeta} \tilde{U}\left[\langle\hat{m}, \nabla u\rangle+\frac{1}{\zeta} \tilde{\theta} u\right]+V^{-} u\right) \mu_{S}+\int_{D(a)}\left(V^{-} t^{a} \nabla_{a} u-u t^{a} \nabla_{a} V^{-}\right) \mu_{a},
\end{aligned}
$$

where $\tilde{U}$ is a smooth function.

Energy estimates. Given a space-like hypersurface $D \subset \Sigma_{a}$, local energy estimates for solutions of the Cauchy problem can be derived in a region

$$
\Delta(a, b)=\{q \in \text { Domain of dependence of } D ; a \leq t(q) \leq b\} .
$$

For any vector field $V$, using the notation introduced earlier, we define energy norms on $D(t)$ and on $M\left(t_{1}, t_{2}\right)$ as

$$
\begin{aligned}
\|V(t)\|_{H^{1}(D(t))}^{2} & =\left\|\nabla_{\hat{t}} V\right\|_{L^{2}(D(t))}^{2}+\sum\left\|\nabla_{\hat{z}_{i}} V\right\|_{L^{2}(D(t))}^{2}+\|V\|_{L^{2}(D(t))}, \\
\left\|V_{t a n}\right\|_{L^{2}\left(M\left(t_{1}, t_{2}\right)\right)}^{2} & =\|\langle\hat{\ell}, V\rangle\|_{L^{2}\left(M\left(t_{1}, t_{2}\right)\right)}^{2}+\sum\left\|\left\langle\hat{e}_{i}, V\right\rangle\right\|_{L^{2}\left(M\left(t_{1}, t_{2}\right)\right)}^{2}, \\
\left\|\nabla_{t a n} V\right\|_{L^{2}\left(M\left(t_{1}, t_{2}\right)\right)}^{2} & =\left\|\nabla_{\hat{\ell}} V\right\|_{L^{2}\left(M\left(t_{1}, t_{2}\right)\right)}^{2}+\sum\left\|\nabla_{\hat{e}_{i}} V\right\|_{L^{2}\left(M\left(t_{1}, t_{2}\right)\right)}^{2}, \\
\|V\|_{L^{\infty, 2}\left(K\left(t_{1}, t_{2}\right)\right)} & =\sup _{t \in\left[t_{1}, t_{2}\right]}\|V(t)\|_{L^{2}(D(t))},
\end{aligned}
$$

where $L^{p}$ and $H^{k}$ denote the standard Lebesgue and Sobolev spaces on $D(t)$ and $M\left(t_{1}, t_{2}\right)$, with measure $\mu_{t}$ and $\mu_{\ell}$ respectively. In a similar manner we define $\|T\|_{H^{1}}$, $\left\|T_{\text {tan }}\right\|_{L^{2}}$, and $\left\|\nabla_{t a n} T\right\|_{L^{2}}$, for any tensor $T$. obtain

To derive energy estimates for equation (2.2), multiply the equation by $X(u)$ to

$$
\nabla^{\alpha}\left[X^{\beta}\left(u_{; \alpha} u_{; \beta}-\frac{1}{2} g_{\alpha \beta} u_{; \nu} u^{; \nu}\right)\right]-X^{\alpha ; \beta}\left[\left(u_{; \alpha} u_{; \beta}-\frac{1}{2} g_{\alpha \beta} u_{; \nu} u^{; \nu}\right)\right]=f X(u)
$$


The above equation can be expressed as

$$
\nabla^{\alpha}\left(X^{\beta} T_{\alpha \beta}\right)-X^{\alpha ; \beta} T_{\alpha \beta}=f X(u),
$$

where the energy-momentum tensor $T_{\alpha \beta}$ is defined as

$$
T_{\alpha \beta}=\partial_{\alpha} u \partial_{\beta} u-\frac{1}{2} g_{\alpha \beta}\left(g^{\mu \nu} \partial_{\mu} u \partial_{\nu} u\right)
$$

For any $p \in \Delta(a, b)$ integrate equation (2.5) on the truncated cone $K(a, t) \subset K(p)$ to obtain

$$
\begin{aligned}
& \int_{D(t)} T(X, \hat{t}) \mu_{t}+\int_{M(a, t)} T(X, \hat{\ell}) \mu_{\ell} \\
= & \int_{D(a)} T(X, \hat{t}) \mu_{a}+\int_{K(a, t)}\left(X^{\alpha ; \beta} T_{\alpha \beta}+f X(u)\right) \mu .
\end{aligned}
$$

Substituting $X=\hat{t}$, the terms in equation (2.8) can be written as

$$
\begin{aligned}
E(u, D(t)) & :=+\int_{D(t)} t^{\alpha} t^{\beta} T_{\alpha \beta} \mu_{t}=\int_{D(t)}\left[\frac{1}{2}|\hat{t}(u)|^{2}+\frac{1}{2} \sum\left|\hat{z}_{i}(u)\right|^{2}\right] \mu_{t}, \\
\operatorname{flux}(u, M(a, t)) & :=\int_{M(a, t)} \ell^{\alpha} t^{\beta} T_{\alpha \beta} \mu_{\ell}=\frac{1}{2} \int_{M(a, t)} \ell^{\alpha}\left(\ell^{\beta}+m^{\beta}\right) T_{\alpha \beta} \mu_{\ell}, \\
& =\frac{1}{2} \int_{M(a, t)}\left[|\hat{\ell}(u)|^{2}+\sum\left|\hat{e}_{i}(u)\right|^{2}\right] \mu_{\ell},
\end{aligned}
$$

where we used the decomposition of $\nabla u$

$$
\begin{array}{ll}
\nabla u=\hat{t}(u) \hat{t}+\sum \hat{z}_{i}(u) \hat{z}_{i} \quad \text { on } D(t), \\
\nabla u=\frac{1}{2} \hat{m}(u) \hat{\ell}+\frac{1}{2} \hat{\ell}(u) \hat{m}+\sum \hat{e}_{i}(u) \hat{e}_{i} \quad \text { on } M(a, t) .
\end{array}
$$

Thus the conservation of energy can be writtten as

$$
E(u, D(t))+\text { flux }(u, M(a, t))=E(u, D(a))+\int_{K(a, t)}\left[t^{\alpha ; \beta} T_{\alpha \beta}+f \hat{t}(u)\right] \mu .
$$

We have

$$
\int_{K(a, t)} t^{\alpha ; \beta} T_{\alpha \beta} \mu \leq C \int_{a}^{t} E(u, D(s)) d s,
$$

so that (2.8) together with Gronwall's lemma implies

$$
\|\nabla u(t)\|_{L^{2}(D(t))}+\left\|\nabla_{t a n} u\right\|_{L^{2}(M(a, t))} \leq C\|\nabla u\|_{L^{2}(D(a))}+\int_{a}^{t}\|f(s)\|_{L^{2}(D(s))} d s .
$$

Here $\|\nabla u(t)\|_{L^{2}(D(t))}$ is defined as $\sqrt{E(u, D(t))}$. On $K(p)=\left\{q \in J^{-}(p) ; a \leq t(q) \leq\right.$ $t(p)\}$ the above equation can be written as

$$
\|\nabla u\|_{L^{\infty, 2}(K(p))}+\left\|\nabla_{t a n} u\right\|_{L^{2}(C(p))} \leq C\left(\|\nabla u(a)\|_{L^{2}(D)}+\mid t(p)-a\|\| f \|_{L^{\infty, 2}(K(p))}\right) .
$$


We can derive similar estimates on $\Delta(a, b)$

$$
\|\nabla u(t)\|_{L^{2}(\Delta(t))}+\left\|\nabla_{t a n} u\right\|_{L^{2}(\Lambda(a, t))} \leq C\|\nabla u(a)\|_{L^{2}(\Delta(a))}+\int_{a}^{t}\|f(s)\|_{L^{2}(\Delta(s))} d s
$$

where

$$
\begin{aligned}
\Delta(\tau) & =\{q \in \Delta(a, b) ; t(q)=\tau\} \\
\Lambda(a, \tau) & =\{q \in \text { null boundary of } \Delta(a, b) ; a \leq t(q) \leq \tau\} .
\end{aligned}
$$

The estimate on $\Delta(a, b)$ combined with the estimate on $C(p)$ implies

$$
\begin{aligned}
& \|\nabla u\|_{L^{\infty, 2}(\Delta(a, b))}+\sup _{p \in \Delta(a, b)}\left\|\nabla_{t a n} u\right\|_{L^{2}(C(p))} \\
\leq & C\left(\|\nabla u(a)\|_{L^{2}(D)}+\mid b-a\|\| f \|_{L^{\infty, 2}(\Delta(a, b))}\right) .
\end{aligned}
$$

where $\|\nabla u\|_{L^{\infty, 2}(\Delta(a, b))}:=\sup _{a \leq t \leq b}\|\nabla u(t)\|_{L^{2}(\Delta(t))}$.

3. The Yang-Mills Equations. Recall that a connection $A$ on $P$, a $G$-principal bundle over $M$, can be expressed in a coordinate patch as

$$
A=A_{\mu}(x) d x^{\mu}, \quad A_{\mu}(x) \in \mathfrak{g}
$$

and the curvature $F$ as

$$
F_{\mu \nu}=\partial_{\mu} A_{v}-\partial_{\nu} A_{\mu}+\left[A_{\mu}, A_{\nu}\right]
$$

We shall assume for simplicity that the elements of $\mathfrak{g}$ are matrices, and that the adjoint action $A d$ of $G$ on $\mathfrak{g}$ corresponds to an appropriate multiplication by matrices. Then the gauge transformations are given by a smooth map $\mathcal{G} \in G$

$$
A_{\mu}^{\prime}=\mathcal{G}^{-1} A_{\mu} \mathcal{G}+\mathcal{G}^{-1} \partial \mathcal{G}
$$

and gauge covariant derivative by $\mathcal{D}_{\mu}=\nabla_{\mu}+\left[A_{\mu}\right.$, ]. This implies

$$
\begin{aligned}
& F_{\mu \nu}^{\prime}=\mathcal{G}^{-1} F_{\mu \nu} \mathcal{G} \\
& \mathcal{D}_{\alpha}^{\prime} F_{\mu \nu}^{\prime}=\mathcal{G}^{-1} \mathcal{D}_{\alpha} F_{\mu \nu} \mathcal{G}
\end{aligned}
$$

The Yang-Mills equations and the Bianchi identity are given by

$$
\begin{gathered}
\mathcal{D}_{\mu} F^{\mu \nu}=F^{\mu \nu}{ }_{; \mu}+\left[A_{\mu}, F^{\mu \nu}\right]=0, \\
\mathcal{D}_{\alpha} F_{\mu \nu}+\mathcal{D}_{\mu} F_{\nu \alpha}+\mathcal{D}_{\nu} F_{\alpha \mu}=0 .
\end{gathered}
$$

From this first order system, we can derive a covariant wave equation for $F$ by differentiating $(3.3 \mathrm{~b})$ with respect to $\mathcal{D}_{\alpha}$ and using $(3.3 \mathrm{a})$ and the commutation relation of covariant derivatives

$$
\mathcal{D}_{\alpha} \mathcal{D}_{\beta} F_{\mu \nu}=\mathcal{D}_{\beta} \mathcal{D}_{\alpha} F_{\mu \nu}-R_{\mu \alpha \beta}^{\gamma} F_{\gamma \nu}-R_{\nu \alpha \beta}^{\gamma} F_{\mu \gamma}+\left[F_{\alpha \beta}, F_{\mu \nu}\right]
$$

where $R$ is the curvature tensor, to obtain

$$
-\square F_{\mu \nu}+2\left[F_{\mu}{ }^{\beta}, F_{\beta \nu}\right]-2 R_{\alpha \mu \nu \beta} F^{\alpha \beta}-R_{\mu \alpha} F_{\nu}^{\alpha}-R_{\nu \alpha} F_{\mu}^{\alpha}=0,
$$


where the covariant wave operator is given by

$$
-\square F_{\mu \nu}:=\mathcal{D}^{\alpha} \mathcal{D}_{\alpha} F_{\mu \nu}=g^{\alpha \beta} F_{\mu \nu ; \alpha \beta}+\left[A^{\alpha}, F_{\mu \nu}\right]_{; \alpha}+\left[A^{\alpha}, F_{\mu \nu ; \alpha}\right]+\left[A^{\alpha},\left[A_{\alpha}, F_{\mu \nu}\right]\right] .
$$

In a manner similar to the wave equation, we can derive estimates for solutions of the Cauchy problem for the Yang-Mills equations, in a geodesically convex neighbourhood, with data given on a space-like hypersurface $D \subset \Sigma_{a}$. As before, define $\Delta(a, b)$ and $K(p)$,

$$
\begin{aligned}
\Delta(a, b) & =\{q \in \text { Domain of dependence of } D ; a \leq t(q) \leq b\}, \\
K(p) & =\left\{q \in J^{-}(p) ; a \leq t(q) \leq t(p)\right\}
\end{aligned}
$$

with $\delta=b-a>0$ small; and denote by $\Lambda(a, b)$ and $C(p)$ the null boundaries of $\Delta(a, b)$ and $K(p)$ respectively. In what follows, we derive a priori estimates for solutions of the Yang-Mills equations in the region $\Delta(a, b)$.

$L^{2}$ estimates. As for the wave equation, we can define an energy-momentum tensor for the Yang-Mills equations,

$$
T_{\alpha \beta}:=F_{\alpha \mu} \cdot F_{\beta}{ }^{\mu}-\frac{1}{4} g_{\alpha \beta} F_{\mu \nu} \cdot F^{\mu \nu}
$$

where $A \cdot B=k(A, B)$, a positive definite Ad-invariant metric on $\mathfrak{g}$. This tensor satisfies $\nabla^{\beta} T_{\alpha \beta}=0$, which implies an a priori $L^{2}$ bound on $F$. To derive this bound integrate $t^{\alpha} T_{\alpha \beta}$ on a truncated cone $K(a, t) \subset K(p)$, to obtain

$$
\int_{D(t)} T(\hat{t}, \hat{t}) \mu_{t}+\int_{M(a, t)} T(\hat{t}, \hat{\ell}) \mu_{\ell}=\int_{D(a)} T(\hat{t}, \hat{t}) \mu_{a}+\int_{K(a, t)} \hat{t}^{\alpha ; \beta} T_{\alpha \beta} \mu
$$

We note that

$$
\begin{aligned}
\int_{D(t)} t^{\alpha} t^{\beta} T_{\alpha \beta} \mu_{t} & =\int_{D(t)}\left[\frac{1}{2} F_{\hat{z}_{i} \hat{t}} \cdot \sum F_{\hat{z}_{i} \hat{t}}+\frac{1}{4} \sum F_{\hat{z}_{i} \hat{z}_{j}} \cdot F_{\hat{z}_{i} \hat{z}_{j}}\right] \mu_{t} \approx\|F(t)\|_{L^{2}(D(t))}^{2} \\
\int_{M(a, t)} \ell^{\alpha} t^{\beta} T_{\alpha \beta} \mu_{\ell} & =\int_{M(a, t)}\left[\frac{1}{2} \sum F_{\hat{e}_{i} \hat{\ell}} \cdot F_{\hat{e}_{i} \hat{\ell}}+\frac{1}{4} \sum F_{\hat{e}_{i} \hat{e}_{j}} \cdot F_{\hat{e}_{i} \hat{e}_{j}}+\frac{1}{8} F_{\hat{\ell}_{\hat{m}}} \cdot F_{\hat{\ell}_{\hat{m}}}\right] \mu_{\ell} \\
& \approx\left\|F_{t a n}\right\|_{L^{2}(M(a, t))}^{2} .
\end{aligned}
$$

It follows that we have the a priori estimate

$$
\|F(t)\|_{L^{2}(D(t))}+\left\|F_{t a n}\right\|_{L^{2}(M(a, t))} \leq C\|F(a)\|_{L^{2}(D(a))} .
$$

If we denote the square root of energy on $D$ by

$$
E_{0}=\|F(a)\|_{L^{2}(D)}
$$

the above energy estimate can be written as

$$
\|F\|_{L^{\infty, 2}(K(p))}+\left\|F_{t a n}\right\|_{L^{2}(C(p))} \leq C E_{0} .
$$

Similarly we derive the following estimate on $\Delta(a, b)$

$$
\|F\|_{L^{\infty, 2}(\Delta(a, b))}+\sup _{p \in \Delta(a, b)}\left\|F_{t a n}\right\|_{L^{2}(C(p))} \leq C E_{0} .
$$


$H^{1}$ estimates. Derivative estimates for $F$ can be derived from equation (3.5) in a manner similar to that of the wave equation: Let $\hat{t}, \hat{z}_{i}$ be an ON basis as described at the end of the introduction, and let $h^{\alpha \beta}$ be the Riemannian metric on $M$ defined by $h^{\alpha \beta}=\hat{t}^{\alpha} \hat{t}^{\beta}+\sum_{i=1}^{3} \hat{z}_{i}^{\alpha} \hat{z}_{i}^{\beta}$. Consider the new "energy-momentum tensor" $T^{\alpha \beta}$ defined as

$$
T^{\alpha \beta}=h^{\mu \nu} h^{\rho \sigma}\left\{k\left(\mathcal{D}^{\alpha} F_{\mu \rho}, \mathcal{D}^{\beta} F_{\nu \sigma}\right)-\frac{1}{2} k\left(\mathcal{D}^{\lambda} F_{\mu \rho}, \mathcal{D}_{\lambda} F_{\nu \sigma}\right) g^{\alpha \beta}\right\}
$$

We have

$$
\begin{aligned}
\nabla_{\alpha}\left(T^{\alpha \beta} \hat{t}_{\beta}\right)= & \nabla_{\alpha}\left(h^{\mu \nu} h^{\rho \sigma} \hat{t}_{\beta}\right)\left\{k\left(\mathcal{D}^{\alpha} F_{\mu \rho}, \mathcal{D}^{\beta} F_{\nu \sigma}\right)-\frac{1}{2} k\left(\mathcal{D}^{\lambda} F_{\mu \rho}, \mathcal{D}^{\lambda} F_{\nu \sigma}\right) g^{\alpha \beta}\right\}+ \\
& h^{\mu \nu} h^{\rho \sigma} \hat{t}_{\beta}\left\{k\left(\mathcal{D}_{\alpha} \mathcal{D}^{\alpha} F_{\mu \rho}, \mathcal{D}^{\beta} F_{\nu \sigma}\right)+k\left(\mathcal{D}^{\alpha} F_{\mu \rho},\left(\mathcal{D}_{\alpha} \mathcal{D}^{\beta}-\mathcal{D}^{\beta} \mathcal{D}_{\alpha}\right) F_{\nu \sigma}\right)\right\}
\end{aligned}
$$

Integrating this equation over $K(a, t)$ and using (3.4) and (3.5) to get rid of second derivatives of $F$ we obtain

$$
\begin{aligned}
& \|\mathcal{D} F(t)\|_{L^{2}(D(t))}^{2}+\left\|\mathcal{D}_{\tan } F\right\|_{L^{2}(M(a, t))}^{2} \\
\leq & \|\mathcal{D} F(a)\|_{L^{2}(D(a))}^{2}+C \int_{K(a, t)}|\mathcal{D} F|\left(|F|^{2}+|F|+|\mathcal{D} F|\right),
\end{aligned}
$$

where $\mathcal{D} F$ denotes the gauge covariant gradient of $F$, and

$$
\left|\mathcal{D}_{\text {tan }} F\right|=\left|\mathcal{D}_{\hat{\ell}} F\right|+\sum\left|\mathcal{D}_{\hat{e}_{i}} F\right| \text {. }
$$

From Gronwall's lemma it then follows

$$
\begin{aligned}
& \|\mathcal{D} F(t)\|_{L^{2}(D(t))}+\left\|\mathcal{D}_{t a n} F\right\|_{L^{2}(M(a, t))} \\
\leq & c\|\mathcal{D} F(a)\|_{L^{2}(D(a))}+C \int_{a}^{t}\left(\|F(s)\|_{L^{4}(D(s))}^{2}+\|F(s)\|_{L^{2}(D(s))}\right) d s .
\end{aligned}
$$

This implies

$$
\begin{aligned}
& \|\mathcal{D} F(t)\|_{L^{2}(D(t))}+\left\|\mathcal{D}_{t a n} F\right\|_{L^{2}(M(a, t))} \\
\leq & C\left(\|\mathcal{D} F(a)\|_{L^{2}(D(a))}+\|F(a)\|_{L^{2}(D(a))}+|t-a| \sup _{q \in K(p)}|F(q)|\right),
\end{aligned}
$$

where $C$ depends on the energy $E_{0}=\|F(a)\|_{L^{2}(D)}$. The above equation is equivalent to

$$
\|D F\|_{L^{\infty, 2}(K(p))}+\left\|\mathcal{D}_{t a n} F\right\|_{L^{2}(C(p))} \leq C\left(\|D F(a)\|_{L^{2}(D(a))}+\delta\|F\|_{L^{\infty}(K(p))}\right) .
$$

Here, as before, $\delta=|b-a|$. Repeating the same argument on $\Delta(a, b)$ we obtain

$$
\|\mathcal{D} F(t)\|_{L^{2}(\Delta(t))}+\left\|\mathcal{D}_{t a n} F\right\|_{L^{2}(\Lambda(a, t))} \leq C_{1}\|D F(a)\|_{L^{2}(D)}+C_{2}|t-a| \sup _{q \in \Delta(a, b)}|F(q)|,
$$

where $\Delta(\tau)=\{q \in \Delta ; t(q)=\tau\}$. Combining the above with equation (3.11) gives

$\|\mathcal{D} F\|_{L^{\infty, 2}(\Delta(a, b))}+\sup _{p \in \Delta(a, b)}\left\|\mathcal{D}_{t a n} F\right\|_{L^{2}(C(p))} \leq C\left(\|\mathcal{D} F(a)\|_{L^{2}(D)}+\delta\|F\|_{L^{\infty}(\Delta(a, b)}\right)$. 
Cronström gauge estimates. To obtain bounds on higher energy norms of $F$, we see from equation (3.12) that we need a pointwise estimate on $F$. This will be done, as in [9], by using the Cronström gauge,

$$
\langle d \gamma(p, q), A(q)\rangle=0 \quad A(p)=0 .
$$

This choice of gauge has the advantage of allowing us to express $A$ in terms of $F$ in a simple manner. Using coordinates that are normal and Minkowskian at $p$ the Cronström gauge is written as

$$
\gamma^{; \alpha}(x) A_{\alpha}(x)=2 x^{\alpha} A_{\alpha}(x)=0, \quad A_{\alpha}(0)=0 .
$$

From equation (3.1) we obtain

$$
\begin{aligned}
x^{\alpha} F_{\alpha \beta}(x) & =x^{\alpha}\left(\partial_{\alpha} A_{\beta}-\partial_{\beta} A_{\alpha}\right) \\
& =x^{\alpha} \partial_{\alpha} A_{\beta}+A_{\beta} .
\end{aligned}
$$

The above equation has the solution

$$
A_{\beta}(x)=\int_{0}^{1} x^{\alpha} F_{\alpha \beta}(s x) s d s .
$$

Estimates of $A$ on $C(p)$. On $C(p), A$ can be estimated in terms of $F$ in the following manner. As before, parameterize $C(p) \backslash p$ by $(\zeta, \omega) \in \mathbb{R}^{+} \times \mathbb{S}^{2}$ where any $q \in C^{-}(p) \backslash p$ can be represented by $q=\exp _{p}(-\zeta \hat{\ell})$. From (3.13) we obtain

$$
\left|\frac{A_{\alpha}(x)}{\zeta}\right| \leq \int_{0}^{1}\left|F_{\alpha \hat{\ell}}(s x)\right| s d s \leq\|F\|_{L^{\infty}(K(p))} .
$$

By integrating the above equation on $C(p)$, we obtain

$$
\left\|\frac{A_{\alpha}}{\zeta}\right\|_{L^{2}(C(p))} \leq C \int_{0}^{1} s^{-3 / 2}\left\|F_{\alpha \hat{\ell}}\right\|_{L^{2}(C(p))} s d s \leq C\left\|F_{t a n}\right\|_{L^{2}(C(p))} \leq C E_{0} .
$$

By integrating equation (3.14) over $S=D(a) \cap C(p)$, we have

$$
\int_{S}\left|A_{\alpha}\right| \mu_{S} \leq C \zeta_{1} \int_{C(p)} \frac{1}{\zeta}\left|F_{\alpha \hat{\ell}}\right| \mu_{\ell} \leq C \zeta_{1}^{3 / 2}\left\|F_{t a n}\right\|_{L^{2}(C(p))} \leq C \zeta_{1}^{3 / 2} E_{0}
$$

where $\zeta_{1}=\max \{\zeta(q) ; q \in C(p)\}$.

Estimates on $D(t)$. The following estimates are obtained from equation (3.13) and (3.14) in a straightforward manner.

$$
\begin{aligned}
\|A(t)\|_{L^{2}(D(t))} & \leq C \zeta_{1}\|F\|_{L^{\infty, 2}(K(p))} \leq C \zeta_{1} E_{0} \\
\|\nabla A(t)\|_{L^{2}(D(t))} & \leq C\left(\zeta_{1}\|\mathcal{D} F\|_{L^{\infty, 2}(K(p))}+\zeta_{1}^{2}\|F\|_{L^{\infty}(K(p))} E_{0}+E_{0}\right)
\end{aligned}
$$

where in the above inequality we used equation (3.14) to substitute $\mathcal{D} F$ for $\nabla F$. 
Pointwise estimates on F. Since $F$ satisfies equation (3.5), we have from the tensorial equivalent of equation (2.3) (cf. [10], Section 5.5, together with the arguments of the proof of Theorem 5.3.3 there) the following representation for $F$

$$
F_{\mu \nu}(p)=\int_{C(p)} H \frac{\kappa(\zeta, \omega)}{\zeta} \mu_{\ell}+\int_{K(p)} H V^{-} \mu+F_{\mu \nu}^{l i n}(p)
$$

where $F_{\mu \nu}^{l i n}$ is a solution of the homogeneous covariant wave equation (3.6), and where

$$
\begin{aligned}
H=2\left[F_{\mu}{ }^{\beta},\right. & \left.F_{\beta \nu}\right]-2 R_{\alpha \mu \nu \beta} F^{\alpha \beta}-R_{\mu \alpha} F_{\nu}{ }^{\alpha}+R_{\nu \alpha} F_{\mu}{ }^{\alpha} \\
& +\left[A^{\alpha}, F_{\mu \nu}\right]_{; \alpha}+\left[A^{\alpha}, F_{\mu \nu ; \alpha}\right]+\left[A^{\alpha},\left[A_{\alpha}, F_{\mu \nu}\right]\right]
\end{aligned}
$$

Here $\kappa$ and $V^{-}$are smooth tensor fields; for notational convenience we have suppressed indices on $H, \kappa$ and $V^{-}$. If $A$ and $F$ are given in the Cronström gauge, so that $A$ is tangential to $C(p)$, we can integrate by parts on the term $\left[A^{\alpha}, F_{\mu \nu}\right]_{; \alpha}$ to rewrite equation (3.19) as

$$
F_{\mu \nu}(p)=\int_{C(p)} \frac{\tilde{H} \kappa(\zeta, \omega)}{\zeta} \mu_{\ell}+\int_{K(p)} H V^{-} \mu+F_{\mu \nu}^{l i n}(p)+\int_{S} \frac{\kappa}{\zeta} m^{\alpha}\left[A_{\alpha}, F_{\mu \nu}\right] \mu_{S} .
$$

where $S=C(p) \cap D(a)$, and

$$
\begin{aligned}
\tilde{H}=2\left[F_{\mu}{ }^{\beta},\right. & \left.F_{\beta \nu}\right]-2 R_{\alpha \mu \nu \beta} F^{\alpha \beta}-R_{\mu \alpha} F_{\nu}{ }^{\alpha}+R_{\nu \alpha} F_{\mu}{ }^{\alpha} \\
& -\kappa_{; \alpha}\left[A^{\alpha}, F_{\mu \nu}\right]+\left[A^{\alpha}, F_{\mu \nu ; \alpha}\right]+\left[A^{\alpha},\left[A_{\alpha}, F_{\mu \nu}\right]\right] .
\end{aligned}
$$

The integral on the surface of the cone in equation (3.20) can be bounded using equation (3.13),

$$
\begin{aligned}
\left\|\frac{1}{\zeta}\left[A^{\alpha}, F_{\mu \nu}\right]\right\|_{L^{1}(C(p))} & \leq C\left\|\frac{A^{\alpha}}{\zeta}\right\|_{L^{1}(C(p))}\|F\|_{L^{\infty}(C(p))} \leq C \sqrt{\delta}\|F\|_{L^{\infty}(K(p))}, \\
\left\|\left[\frac{1}{\zeta} A^{\alpha}, F_{\mu \nu ; \alpha}\right]\right\|_{L^{1}(C(p))} & \leq C\left\|\mathcal{D}_{t a n} F\right\|_{L^{2}(C(p))}, \\
\left\|\frac{1}{\zeta}\left[A_{\alpha},\left[A^{\alpha}, F_{\mu \nu}\right]\right]\right\|_{L^{1}(C(p))} & \leq C \delta\|F\|_{L^{\infty}(K(p))}, \\
\left\|\frac{1}{\zeta}\left[F_{\mu}{ }^{\beta}, F_{\beta \nu}\right]\right\|_{L^{1}(C(p))} & \leq C\|F\|_{L^{\infty}(K(p))}\left\|\frac{F}{\zeta}\right\|_{L^{1}(C(p))} \leq C \sqrt{\delta}\|F\|_{L^{\infty}(K(p))},
\end{aligned}
$$

where in the last inequality we used the observation of Eardley and Moncrief [9], that $\left[F_{\mu}{ }^{\beta}, F_{\beta \nu}\right]$ consists of tangential terms only (i.e. $\left[F_{\hat{\ell} \hat{e_{i}}}, F_{\hat{\ell} \hat{e_{i}}}\right]$ and $\left[F_{\hat{\hat{e}_{i}} \hat{\hat{e}_{j}}}, F_{\hat{\hat{e}_{i}} \hat{\hat{e}_{j}}}\right]$ ). Similarly the integral over the solid cone can be estimated using (3.17) and (3.18) to obtain

$$
\begin{aligned}
\left|\int_{K(p)} V^{-} H \mu\right| \leq & C\left(\|A\|_{L^{2}(K(p))}\|\mathcal{D} F\|_{L^{2}(K(p))}\right. \\
& \left.+\|A\|_{L^{\infty}(K(p))}\|A\|_{L^{2}(K(p))}\|F\|_{L^{2}(K(p))}+\delta E_{0}\right) \\
\leq & C\left(\delta\|\mathcal{D} F\|_{L^{\infty, 2} K(p)}+\delta^{2}\|F\|_{L^{\infty}(K(p))}+\delta E_{0}\right)
\end{aligned}
$$


Since the curvature is bounded on bounded sets, then the first two terms in equation (3.20) can be bounded by a constant times

$$
E_{0}+\|\mathcal{D} F\|_{L^{\infty, 2}(K(p))}+\left\|\mathcal{D}_{\tan } F\right\|_{L^{2}(C(p))}+\sqrt{\delta}\|F\|_{L^{\infty}(K(p))}
$$

The remaining terms, $F_{\mu \nu}^{l i n}(p)$ together with the last term from eq. (3.20), consist of the following expressions

$$
\begin{aligned}
& \int_{D(a)}\left(V^{-} t^{\alpha} \nabla_{\alpha} F_{\mu \nu}-F_{\mu \nu} V^{-}\right) \mu_{a} \\
= & \int_{D(a)}\left(V^{-} t^{\alpha} \mathcal{D}_{\alpha} F_{\mu \nu}-F_{\mu \nu} V^{-}-V^{-}\left[A^{\alpha}, F_{\mu \nu}\right]\right) \mu_{a}, \\
& \int_{S} \frac{1}{\zeta} \tilde{U}\left(m^{\alpha} \nabla_{\alpha} F_{\mu \nu}+\frac{1}{\zeta} \tilde{\theta} F_{\mu \nu}\right) \mu_{S} \\
= & \int_{S} \frac{1}{\zeta} \tilde{U}\left(m^{\alpha} \mathcal{D}_{\alpha} F_{\mu \nu}+\frac{1}{\zeta} \tilde{\theta} F_{\mu \nu}\right) \mu_{S}-\int_{S} \frac{1}{\zeta} \tilde{U} m^{\alpha}\left[A_{\alpha}, F_{\mu \nu}\right] \mu_{S}, \\
& \int_{S} V^{-} F_{\mu \nu} \mu_{S}, \\
& \int_{S} \frac{\kappa}{\zeta} m^{\alpha}\left[A_{\alpha}, F_{\mu \nu}\right] \mu_{S} .
\end{aligned}
$$

Here $\tilde{U}$ and $\tilde{\theta}$ are smooth tensor fields (with indices suppressed). The terms in $(3.22 \mathrm{~b})$ and $(3.22 \mathrm{c})$ can be bounded by a constant by

$$
\|\mathcal{D} F(a)\|_{L^{2}(D(a))}+\|F(a)\|_{L^{2}(D(a))} .
$$

By the divergence theorem, the terms in $(3.22 \mathrm{c})$ and $(3.22 \mathrm{~d})$ can be bounded by a constant times

$$
\begin{aligned}
& \frac{1}{r_{1}} \int_{D(a)}\left(|\nabla \mathcal{D} F(a)|+\frac{1}{r}(|\nabla F(a)|+|\mathcal{D} F(a)|)\right. \\
+ & \left.\frac{1}{r^{2}}|F(a)|\right) \mu_{a}+\frac{1}{r_{1}}\|F\|_{L^{\infty}(K(p))} \int_{S}|A| d \mu_{S},
\end{aligned}
$$

where in normal coordinates centered at $p, r=|\mathrm{x}|, r_{1}=\min \{\zeta(q) ; q \in S\}$ and $r_{2}=\max \{\zeta(q) ; q \in S\}$. The ratio $\frac{r_{2}}{r_{1}}$ is bounded by a constant depending on $p$.

The first term in equation (3.24) can be bounded by a constant times

$$
\frac{1}{r_{1}} \int_{D(a)}|\nabla \mathcal{D} F(a)| \mu_{a} \leq \frac{1}{r_{1}} \int_{D(a)}(|\mathcal{D D} F(a)|+C|A||\mathcal{D} F|) \mu_{a} \leq C E_{2}(a)
$$

where we have used $\int_{D(a)} \mu_{a} \leq C r_{1}^{3}$, and where we have defined

$$
E_{2}(\tau)=\|\mathcal{D D} F(t)\|_{L^{2}(\Delta(\tau))}+\|\mathcal{D} F(t)\|_{L^{2}(\Delta(\tau))}+\|F(t)\|_{L^{2}(\Delta(\tau))}
$$


The second term in equation (3.24) can be bounded by a constant times

$$
\begin{aligned}
& \frac{1}{r_{1}} \int_{D(a)} \frac{1}{r}(|\nabla F|+|\mathcal{D} F|) \mu_{a} \\
\leq & \frac{1}{r_{1}} \int_{D(a)} \frac{1}{r}|\mathcal{D} F(a)| \mu_{a}+C E_{0} \\
\leq & \frac{1}{r_{1}} \int_{D(a)}(|\nabla \mathcal{D} F(a)|+C|\mathcal{D} F(a)|) \mu_{a}+C E_{0} \\
\leq & C\left(\frac{1}{r_{1}} \int_{D(a)}(|\mathcal{D D} F(a)|+|A \mathcal{D} F(a)|+|\mathcal{D} F(a)|) \mu_{a}+E_{0}\right) \\
\leq & C E_{2}(a) .
\end{aligned}
$$

The third term in equation (3.24) can be bounded by a constant times

$$
\frac{1}{r_{1}} \int_{D(a)} \frac{1}{r^{2}}|F(a)| \mu_{a} \leq \frac{1}{r_{1}} \int_{D(a)} \frac{1}{r}|\mathcal{D} F(a)| \mu_{a}+C E_{0} \leq C E_{2}(a) .
$$

From equation (3.16) the last term in equation (3.24) can be bounded by

$$
C\left(r_{2}\right)^{\frac{1}{2}} E_{0}\|F\|_{L^{\infty}(K(p))} \cdot
$$

Equations (3.21) and (3.24) imply

$$
|F(p)| \leq C\left(E_{2}(a)+\left\|\mathcal{D}_{\tan } F\right\|_{L^{2}(C(p))}+\delta^{1 / 2}\|F\|_{L^{\infty}(K(p))}\right) .
$$

Since all of the terms in the above estimate are gauge invariant, by (3.2) the above inequality remains valid in any gauge. By taking sup over $\Delta(a, b)$ we obtain

$$
\|F\|_{L^{\infty}(\Delta(a, b))} \leq C\left(E_{2}(a)+\sup _{p \in \Delta(a, b)}\left\|\mathcal{D}_{t a n} F\right\|_{L^{2}(C(p))}+\delta^{1 / 2}\|F\|_{L^{\infty}(\Delta(a, b))}\right) .
$$

By choosing $\delta$ small, depending on the energy $E_{0}$ and $a$ only, we obtain from the above equation

$$
\|F\|_{L^{\infty}(\Delta(a, b))} \leq C\left(E_{2}(a)+\sup _{p \in \Delta(a, b)}\left\|\mathcal{D}_{t a n} F\right\|_{L^{2}(C(p))}\right) .
$$

$H^{2}$ bound on $F$. From the energy inequality (3.12) and inequality (3.25) we have

$$
\begin{aligned}
& \|\mathcal{D} F\|_{L^{\infty, 2}(\Delta(a, b))}+\sup _{p \in \Delta(a, b)}\left\|\mathcal{D}_{t a n} F\right\|_{L^{2}(C(p))} \\
\leq & C\left(\|\mathcal{D} F(a)\|_{L^{2}(D(a))}+\delta\left(E_{2}(a)+\left\|\mathcal{D}_{t a n} F\right\|_{L^{2}(C(p))}\right)\right) .
\end{aligned}
$$

By taking $\delta$ small enough, depending on the energy $E_{0}$ and $a$ only, we obtain

$$
\|\mathcal{D} F\|_{L^{\infty, 2}(\Delta(a, b))}+\sup _{p \in \Delta(a, b)}\left\|\mathcal{D}_{t a n} F\right\|_{L^{2}(C(p))} \leq C E_{2}(a) .
$$


Taking a space-time and gauge covariant derivative of equation (3.5) we obtain

$$
\begin{aligned}
\mathcal{D}^{\alpha} \mathcal{D}_{\alpha} \mathcal{D}_{\sigma} F_{\mu \nu}= & -\mathcal{D}_{\sigma}\left\{2\left[F_{\mu}{ }^{\beta}, F_{\beta \nu}\right]-2 R_{\alpha \mu \nu \beta} F^{\alpha \beta}-R_{\mu \alpha} F_{\nu}^{\alpha}-R_{\nu \alpha} F_{\mu}{ }^{\alpha}\right\} \\
& +\left[\mathcal{D}^{\alpha} \mathcal{D}_{\alpha}, \mathcal{D}_{\sigma}\right] F_{\mu \nu} .
\end{aligned}
$$

The right-hand-side of this equation can be estimated as

$$
C(|F|+|\mathcal{D} F|+|F||\mathcal{D} F|) .
$$

We can now repeat the argument leading to (3.10) with the energy-momentum tensor (3.9) replaced by

$$
T^{\alpha \beta}=h^{\psi \phi} h^{\mu \nu} h^{\rho \sigma}\left\{k\left(\mathcal{D}^{\alpha} \mathcal{D}_{\psi} F_{\mu \rho}, \mathcal{D}^{\beta} \mathcal{D}_{\phi} F_{\nu \sigma}\right)-\frac{1}{2} k\left(\mathcal{D}^{\lambda} \mathcal{D}_{\psi} F_{\mu \rho}, \mathcal{D}_{\lambda} \mathcal{D}_{\phi} F_{\nu \sigma}\right) g^{\alpha \beta}\right\}
$$

to obtain

$$
E_{2}(t) \leq C\left(E_{2}(a)+\delta\|\mathcal{D} F\|_{L^{\infty, 2}(\Delta(a, b))}\left(1+\|F\|_{L^{\infty}(\Delta(a, b))}\right)\right) .
$$

From equations (3.25)-(3.26) we conclude

$$
E_{2}(t) \leq C\left(a, E_{2}(a)\right)
$$

for all $a \leq t \leq b$. Since the size of the cone $\delta$ depends only on $a$ and $E_{0}$, which is bounded in terms of the intial data we conclude that for any point $p \in M$ and compact hypersurface $\mathcal{S}(p) \subset\{q \in M ; t(q)=t(p)\}$

$$
\|\mathcal{D D} F\|_{L^{2}(\mathcal{S}(p))}+\|\mathcal{D} F\|_{L^{2}(\mathcal{S}(p))} \leq C(\mathcal{S}(p))\left\|F^{i n i}\right\|_{H^{2}}
$$

where $F^{i n i}$ corresponds to the initial data for $F$.

4. Global existence. Let $\mathcal{O}$ be a coordinate patch on $\Sigma_{t}$, using the flow of $\nabla t$ on $M$ we can extend the coordinates $x^{i}$ on $\mathcal{O}$ to coordinates $\left(t, x^{i}\right)=\left(x^{0}, x^{i}\right)$ on $\mathbb{R} \times \mathcal{O}$. In this coordinate system the metric takes the form

$$
g_{\mu \nu} d x^{\mu} d x^{\nu}=-\alpha^{2} d t^{2}+g_{i j} d x^{i} d x^{j}
$$

with some function $\alpha>0$. In all our considerations below we shall only use coordinate systems of this type. While this is clearly not necessary for our analysis, it simplifies some of the calculations involved.

Let $\Omega \subset M$ be an open set. We shall consider connections on $P \mid \Omega$ with the following differentiability properties: We shall assume that $\Omega$ can be covered by coordinate patches $\Omega_{i}$ with coordinates $\left(t, x^{i}\right) \in I \times \mathcal{U}_{i}$ together with local trivialisations of $P$ over $\Omega_{i}$, such that the coordinate components $A_{\mu}$ of the connection are in $C\left(I ; H^{k+1}\left(\mathcal{U}_{i}\right)\right) \cap C^{1}\left(I ; H^{k}\left(\mathcal{U}_{i}\right)\right)$, for some $k \geq 2$.

Consider a trivialisation of $P$ over a coordinate patch $\mathcal{U}$ of the form $I \times \mathcal{O}$, where $I$ is a time interval, such that the hypersurfaces $\Sigma_{t}$ are given by the equation $x^{0}=t$. For the purposes of this section it will be convenient to impose the temporal gauge condition

$$
A_{0}=0 \text {, }
$$


and to define

$$
\begin{aligned}
G_{\mu \nu} & =F_{\mu \nu}-\partial_{\mu} A_{\nu}+\partial_{\nu} A_{\mu}-\left[A_{\mu}, A_{\nu}\right] \\
B^{\lambda} & =\epsilon^{\lambda \alpha \beta \gamma} \mathcal{D}_{\alpha} F_{\beta \gamma} \\
C^{\lambda} & =\mathcal{D}_{\alpha} F^{\alpha \lambda}
\end{aligned}
$$

In the temporal gauge, the $F_{0 i}$ component of eq. (3.1) reduces to

$$
\partial_{t} A_{i}=F_{0 i}
$$

To establish local in time existence of solutions of the Cauchy problem we shall be solving for $A_{i}, F_{i j}$, and $F_{0 i}$ using equation (4.4) together with the equations obtained by setting $B^{i}=C^{i}=0$ :

$$
\begin{aligned}
\mathcal{D}_{t} F_{i j} & =\mathcal{D}_{j} F_{i 0}-\mathcal{D}_{i} F_{j 0} \\
\mathcal{D}_{t} F^{0 i} & =\mathcal{D}_{j} F^{j i}
\end{aligned}
$$

Let us note, that when eqs. (4.4)-(4.6) hold throughout $\mathcal{U}$, and when $G_{\mu \nu}$ defined in eq. (4.3a) vanishes at one value of $t$, then $G_{\mu \nu}$ will vanish throughout $\mathcal{U}$ (and consequently we shall have $B^{\alpha}=0$ throughout $\mathcal{U}$ ). Indeed, the time derivative of the right-hand-side of eq. (4.3a) with $\mu \nu=i j$ vanishes when eqs. (4.4)-(4.5) hold. It then follows that $F_{\mu \nu}$ is the curvature field of the connection $A_{\mu}$. The vanishing of $B^{\alpha}$ is then simply the Bianchi identity. It is important to note that this will be true regardless of whether or not the Yang-Mills constraint equation $C^{0}=0$ holds.

Let us start with a result that involves Lorentzian metrics on $M=\mathbb{R}^{3+1}$. In that case every principal bundle is trivial so that both the connection and its curvature can be considered as fields defined on $M$. We have the following (which, again, holds irrespective of the vanishing of $C^{0}$ ):

Proposition 4.1. Let $g_{\mu \nu}$ be a smooth Lorentzian metric on $\mathbb{R}^{3+1}$ such that the level sets of $t=x^{0}$ are Cauchy surfaces. Consider two $\mathfrak{g}$-valued one-forms $A_{i}^{D}$ and $E_{i}$ defined on $\mathbb{R}^{3} \approx\{t=0\} \subset \mathbb{R}^{3+1}$, with $\left(A_{i}^{D}, E_{i}\right) \in H_{\mathrm{loc}}^{k+1}\left(\mathbb{R}^{3}\right) \times H_{\text {loc }}^{k}\left(\mathbb{R}^{3}\right)$. Then

1. Suppose that $k \geq 4$. There exists a $\mathfrak{g}$-valued one form $A_{\mu}$ defined on $\mathbb{R}^{3+1}$ such that the field $F_{\mu \nu}$ defined by eq. (4.3a) with $G_{\mu \nu}=0$ is the unique solution of equations (4.4)-(4.6), satisfying $\left.F_{0 i}\right|_{t=0}=E_{i},\left.A_{i}\right|_{t=0}=A_{i}^{D}$, and $A_{0}=0$.

2. Suppose that $k \geq 2$ and that $\mathcal{D}_{j} E^{j}=0$ on a neighbourhood of a coordinate ball $D \equiv B(R) \subset\{t=0\}$. Assume moreover that the interior of the future domain of dependence int $\Delta^{+}(D)$ of $D$ has the property that for all $\tau$ its sections int $\Delta^{+}(D) \cap\{t=\tau\}$ are diffeomorphic (as manifolds with boundary) to coordinate balls, when not empty. Then there exists a $\mathfrak{g}$-valued one form $A_{\mu}$ defined on a neighbourhood of $\Delta^{+}(D)$ and satisfying the Yang-Mills equations in int $\Delta^{+}(D)$.

Proof. The proof of the first assertion is a straightforward consequence of the $a$ priori estimates of Section 3, we shall give some details for completeness ${ }^{5}$. Let $B(R)$ be a coordinate ball of radius $R$ in $\{t=0\}$, multiplying the initial data $\left(A_{i}^{D}, E_{i}\right)$ by a cut-off function we obtain initial data $\left(A_{i}^{D, R}, E_{i}^{R}\right)$ which coincide with $\left(A_{i}^{D}, E_{i}\right)$ on $B(R)$ and which vanish outside $B(2 R)$. Modifying the metric in an appropriate way

\footnotetext{
${ }^{4}$ Missing condition inserted.

${ }^{5}$ The idea of allowing a source term $c_{\mu \nu}$ in eq. (4.12) below is due to [12].
} 
we also obtain a metric $g_{\mu \nu}^{R}$ which coincides with $g_{\mu \nu}$ in the domain of dependence $\Delta(B(R))$ of $B(R)$ and is the standard flat Minkowski metric outside of $I \times B(2 R)$, where $I \subset \mathbb{R}$ is a compact time interval.

Now the system of equations (4.4)-(4.6) is symmetric hyperbolic, which is easily checked as follows: Introduce $E_{i}=F_{i 0}, H_{i}=\epsilon_{i}{ }^{j k} F_{j k} / 2$, where $\epsilon_{i j k}=\sqrt{\operatorname{det} g_{i j}} \partial_{i} \vee$ $\partial_{j} \vee \partial_{k} \vee d x^{1} \wedge d x^{2} \wedge d x^{3}$. Eqs. (4.4)-(4.6) can be rewritten as

$$
\begin{aligned}
g^{i j} \partial_{t} A_{j} & =\text { l.o. } \\
\alpha^{-2} g^{i j} \partial_{t} E_{j} & =\epsilon^{i j k} \partial_{j} H_{k}+\text { l.o. }, \\
g^{i j} \partial_{t} H_{j} & =-\epsilon^{i j k} \partial_{j} E_{k}+\text { l.o. }
\end{aligned}
$$

where $\alpha$ is the "lapse" function appearing in (4.1), and "l.o." denotes terms which do not involve derivatives of $A_{i}, E_{i}$ and $H_{i}$. Set $u=\left(A_{i}, E_{i}, H_{i}\right)$ and

$$
L^{0}=\left(\begin{array}{ccc}
g^{i j} & 0 & 0 \\
0 & \alpha^{-2} g^{i j} & 0 \\
0 & 0 & g^{i j}
\end{array}\right)
$$

It follows that (4.7)-(4.9) is of the form

$$
L^{0}(t, x) \partial_{t} u+L^{i}(t, x) \partial_{i} u=M(t, x, u)
$$

with symmetric matrices $L^{\mu}$ (cf. e.g. [15, p. 199]).

Since equations (4.4)-(4.6) form a semilinear symmetric hyperbolic system, we have from standard energy estimates that the modified Cauchy problem has a local solution in $C\left(\left[-T_{1}, T_{2}\right], H^{k}\left(\mathbb{R}^{n}\right)\right)$, provided $k>\frac{n}{2}$. (See, for example ${ }^{6}$, Volume III, Theorem 1.2, p. 362 and Proposition 2.1, page 370 of [17].)

Consider the field $C^{\lambda}$ defined by eq. (4.3c). We have from equation (4.6) $C^{i}=0$, and from the identity $\mathcal{D}_{\alpha} C^{\alpha}=0$ we have

$$
\partial_{t}\left(\sqrt{-\operatorname{det}} g C^{0}\right)=0 \Rightarrow C^{\lambda}=\delta_{0}^{\lambda} a, \quad a=C^{0}(t=0) / \sqrt{-\operatorname{det}} g .
$$

This shows that $a$ is uniformly bounded on $\mathbb{R}^{3+1}$. The modified curvature tensor $F^{R}$ of $A^{R}$ satisfies the equation

$$
-\square F_{\mu \nu}+2\left[F_{\mu}{ }^{\beta}, F_{\beta \nu}\right]-2 R_{\alpha \mu \nu \beta} F^{\alpha \beta}-R_{\mu \alpha} F_{\nu}^{\alpha}-R_{\nu \alpha} F_{\mu}{ }^{\alpha}=c_{\mu \nu},
$$

where $c_{\mu \nu} \equiv \mathcal{D}_{\mu} C_{\nu}-\mathcal{D}_{\nu} C_{\mu}$ is globally controlled in the $L^{\infty}$ norm (all the $C^{k}$ norms if the initial data are smooth). Equation (4.12) differs from eq. (3.5) of Section 3 only by the $c_{\mu \nu}$ term. Moreover, under the gauge transformation $A_{\mu} \rightarrow \mathcal{G}^{-1} \partial_{\mu} \mathcal{G}+\mathcal{G}^{-1} A_{\mu} \mathcal{G}$, $F_{\mu \nu} \rightarrow \mathcal{G}^{-1} F_{\mu \nu} \mathcal{G}$, eq. (4.12) will be transformed to the same equation with $c_{\mu \nu}$ replaced by $\mathcal{G}^{-1} c_{\mu \nu} \mathcal{G}$. The a priori estimates derived in Section 3 carry over to the above equation when $k \geq 4$ (and, hence, $c_{\mu \nu} \in L_{\text {loc }}^{\infty}$ ) and thus imply global existence of solutions for the modified problem. Point 1 follows now from the fact that the solutions of equations (4.4)-(4.6) on $\Delta(B(R))$ are uniquely determined by their initial data on $B(R)$, so that for $R_{1}>R$ we have $\left(\left.F^{R_{1}}\right|_{\Delta(B(R))},\left.A^{R_{1}}\right|_{\Delta(B(R))}\right)=$ $\left(\left.F^{R}\right|_{\Delta(B(R))},\left.A^{R}\right|_{\Delta(B(R))}\right)$. One can therefore patch together all the $\left(F^{R}, A^{R}\right)$ 's to obtain a globally defined solution on $\mathbb{R}^{3+1}$.

\footnotetext{
6 Since equations (4.4)-(4.6) are semilinear it is easy to see, by a simple modification of the arguments given in [17], that the differentiability threshold $k>n / 2+1$ imposed in [17] can be lowered, in our setting, to $k>n / 2$.
} 
To prove point 2, note that to obtain the $H^{2}$ estimates of Section 3 , one has to be able to perform a gauge transformation to the Cronström gauge. This requires some sufficient degree of differentiability of the fields, which might not be satisfied for $k=2$. Further, the estimates used in the proof of point 1 fail to hold globally with $k=2$, because of the potential lack of uniform boundedness of the $c_{\mu \nu}$ term. To overcome those problems, consider a sequence of smooth initial data $\left(A_{i}^{D, R, n}, \hat{E}_{i}^{R, n}\right)$ supported in the ball $B(2 R)$ which converge to $\left(A_{i}^{D, R}, E_{i}^{R}\right)$ in $H^{k+1}\left(\mathbb{R}^{3}\right) \times H^{k}\left(\mathbb{R}^{3}\right)$. By point 1 we have a corresponding sequence of solutions of eqs. (4.4)-(4.6) defined on $\mathbb{R}^{3+1}$. Because the $H^{2}$ estimates of Section 3 in $\Delta(B(R))$, as adapted to include a supplementary $c_{\mu \nu}$ term in eq. (3.5), involve only the $H^{2}(B(R))$ norm of $F_{\mu \nu}(t=0)$ and the $L^{\infty}(B(R))$ norm of $c_{\mu \nu}$, they will continue to hold in $\Delta(B(R))$ when passing to the limit if we show that we can choose an approximating sequence for which the norms $\left\|c_{\mu \nu}\right\|_{L^{\infty}(B(R))} \leq 2\left\|\mathcal{D}_{i} E^{i}\right\|_{C^{1}(B(R))}$ have a bound independent of $n$. To obtain such a sequence, let the $\mathfrak{g}$-algebra valued field $\phi^{R, n}$ be obtained as a solution of the equation

$$
\mathcal{D}^{i}\left(\mathcal{D}_{i} \phi^{R, n}+\hat{E}_{i}^{R, n}\right)=\psi_{1 / n} * \mathcal{D}^{i} E_{i}^{R} .
$$

Here $\psi_{1 / n} * f$ denotes the convolution of a function $f$ with a Friedrichs mollifier. As discussed in [2, Section 5], eq. (4.13) has a unique solution in an appropriate weighted Sobolev space. Note that $\psi_{1 / n} * \mathcal{D}^{i} E_{i}^{R}$ converges to $\mathcal{D}^{i} E_{i}^{R}=0$ on $B(R)$ in the $C^{1}(B(R))$ norm, since $E_{i}$ coincides with $E_{i}^{R}$ in a neighbourhood of $B(R)$. Moreover both $\mathcal{D}^{i} \hat{E}_{i}^{R, n}$ and $\psi_{1 / n} * \mathcal{D}^{i} E_{i}^{R}$ converge to $\mathcal{D}^{i} E_{i}^{R}$ on $\mathbb{R}^{3}$ in the $H^{k-1}\left(\mathbb{R}^{3}\right)$ norm. It follows that $\phi^{R, n}$ converges to zero in $H^{k+1}(B(2 R))$. Setting $E_{i}^{R, n}=\mathcal{D}_{i} \phi^{R, n}+\hat{E}_{i}^{R, n}$, the sequence $\left(A_{i}^{D, R, n}, E_{i}^{R, n}\right)$ will have all the desired properties. To finish the proof of point 2, note that from eq. (4.11) one has that $C^{\mu}=0$ on $\mathbb{R} \times D$. Since $\Delta(D) \subset \mathbb{R} \times D$ the result follows. $\square$

Proof of Theorem 1.1. Consider the collection $\mathcal{P}$ of open subsets $\Omega$ of $M$ with the following properties:

1. $\Omega$ is globally hyperbolic with Cauchy surface $\Sigma$;

2. $\left.P\right|_{\Omega}$ carries a connection which is a Yang-Mills development of the initial data $\left(A^{\Sigma}, E\right)$;

3. $\Omega$ is covered by coordinate patches $\Omega_{i}$ with coordinates $\left(t, x^{i}\right) \in I \times \mathcal{U}_{i}$ together with local trivialisations of $P$ over $\Omega_{i}$, such that the coordinate components $A_{\mu}$ of the connection are in $C\left(I ; H^{k+1}\left(\mathcal{U}_{i}\right)\right) \cap C^{1}\left(I ; H^{k}\left(\mathcal{U}_{i}\right)\right)$, with $A_{0}=0$.

By Proposition 4.1 together with a standard patching procedure $\mathcal{P}$ is not empty. $\mathcal{P}$ is directed by inclusion, and by the Kuratowski-Zorn lemma there exists a maximal element $\widetilde{\Omega}$ in $\mathcal{P}$. Suppose that $\partial \widetilde{\Omega}$ is not empty, by global hyperbolicity of $M$ and of $\widetilde{\Omega}$, changing time orientation if necessary, there exists a point $p \in \partial \widetilde{\Omega}$ with the property that $J^{-}(p) \cap \partial \widetilde{\Omega}=\{p\}$ (cf. e.g. [5, 7]; here we follow the standard notation [13] in which $J^{-}(p)$ consists of the set of points causally related to $p$, and lying to the past of $p$ ). Choose $\epsilon$ small enough so that the set $K=J^{-}(p) \cap \Sigma_{t(p)-\epsilon}$ is covered by a single coordinate system. Since $\partial \widetilde{\Omega}$ is closed we can find a cover of $K$ by open sets $\mathcal{O}_{p}, p \in K$, such that $\partial \widetilde{\Omega} \cap \overline{\mathcal{O}}_{p}=\emptyset$. (Here $\overline{\mathcal{O}}$ denotes the closure of the set $\mathcal{O}$.) By compactness of $K$ a finite number of the $\mathcal{O}_{p}$ 's can be chosen, $p=p_{i}, i=1, \ldots, N$. Set $\widehat{\mathcal{O}}=\cup_{i=1}^{N} \mathcal{O}_{p_{i}}$. Without loss of generality we can assume that the domain of dependence $\Delta(\widehat{\mathcal{O}})$ of $\widehat{\mathcal{O}}$ is conditionally compact and lies within a single coordinate patch. It should be clear that $p$ lies in the interior of $\Delta(\widehat{\mathcal{O}})$. Let $q$ be any point in the interior of $\Delta(\widehat{\mathcal{O}}) \cap J^{+}(p)$, where $J^{+}(p)$ is the set of points in the causal future of $p$. Let $\mathcal{O}$ be the interior (relative 
to the topology of $\Sigma_{t(p)-\epsilon}$ ) of $J^{-}(q) \cap \Sigma_{t(p)-\epsilon}$. We have $\mathcal{O} \subset \widehat{\mathcal{O}}$, hence $\overline{\mathcal{O}} \cap \partial \widetilde{\Omega}=\emptyset$. Now the interior of the future domain of dependence $\Delta^{+}(\mathcal{O})$ of $\mathcal{O}$ coincides with the interior of $J^{-}(q) \cap J^{+}\left(\Sigma_{t(p)-\epsilon}\right)$, so that (decreasing $\epsilon$ if necessary) $\Delta^{+}(\mathcal{O}) \cap \Sigma_{t}$ is diffeomorphic to a three-dimensional coordinate ball for $t \in(t(p)-\epsilon, t(q))$. We can extend the metric $\left.g_{\mu \nu}\right|_{\Delta(\mathcal{O})}$ from $\Delta(\mathcal{O})$ to a smooth globally hyperbolic metric defined on $\mathbb{R}^{3+1}$ in any way. Similarly we can extend the Yang-Mills initial data induced on $\mathcal{O}$ by the Yang-Mills field on $\widetilde{\Omega}$ in any way. By point 2 of Proposition 4.1 there exists a global solution $\widehat{A}$ of equations (4.4)-(4.6) on $\mathbb{R}^{3+1}$. By uniqueness of solutions of equations (4.4)-(4.6) in domains of dependence, the connection $\widehat{A}$ coincides with $A$ on $\Delta(\mathcal{O}) \cap \widetilde{\Omega}$. Therefore one can extend the connection $A$ from $\left.P\right|_{\widetilde{\Omega}}$ to a connection field on $\Delta(\mathcal{O}) \cup \widetilde{\Omega}$, the extended connection being a Yang-Mills development of the initial data $\left(A^{\Sigma}, E\right)$. This, however, contradicts maximality of $\widetilde{\Omega}$ so that $\widetilde{\Omega}=M$, and Theorem 1.1 is proved.

\section{REFERENCES}

[1] Y. Choquet-Bruhat, Global solutions of Yang-Mills equations on anti-de Sitter spacetime, Class. Quantum Grav., 6 (1989), pp. 1781-1789.

[2] —, Yang-Mills fields on Lorentzian manifolds, in Mechanics, analysis and geometry: 200 years after Lagrange, North-Holland Delta Ser., North-Holland, Amsterdam, 1991, pp. 289313.

[3] — Y Yang-Mills-Higgs fields in three space time dimensions, Mém. Soc. Math. France (N.S.), 46 (1991), pp. 73-97. Analyse globale et physique mathématique (Lyon, 1989).

[4] Y. Choquet-Bruhat and D. Christodoulou, Existence of global solutions of the Yang-Mills, Higgs and spinor field equations in $3+1$ dimensions, Ann. Sci. École Norm. Sup, 14 (1981), pp. 481-506.

[5] Y. Choquet-Bruhat AND R. Geroch, Global aspects of the Cauchy problem in general relativity, Comm. Math. Phys., 14 (1969), pp. 329-335.

[6] Y. Choquet-Bruhat and I. E. Segal, Solution globale des équations de Yang-Mills sur l'univers d'Einstein, C. R. Acad. Sci. Paris Sr. I Math., 294 (1982), pp. 225-230.

[7] Y. Choquet-Bruhat and J. York, The Cauchy problem, in General Relativity, A. Held, ed., Plenum Press, New York, 1980.

[8] D. Christodoulou, Solutions globales des équations de Yang et Mills, C. R. Acad. Sci. Paris Sr. I Math., 293 (1981), pp. 139-141.

[9] D. EARDLEY AND V. MONCRIEF, The global existence of Yang-Mills-Higgs fields in 4dimensional Minkowski space. II. Completion of proof, Comm. Math. Phys., 83 (82), pp. 193-212.

[10] F. G. FRIEDlander, The wave equation on a curved space-time, Cambridge University Press, Cambridge, 1975. Cambridge Monographs on Mathematical Physics, No. 2.

[11] R. Geroch, Domain of dependence, Jour. Math. Phys., 11 (70), pp. 437-449.

[12] M. V. Goganov And L. V. KapitanskǏ, Global solvability of the Cauchy problem for YangMills-Higgs equations, Zap. Nauchn. Sem. Leningrad. Otdel. Mat. Inst. Steklov. (LOMI), 147 (1985), pp. 18-48, 203-204. Boundary value problems of mathematical physics and related problems in the theory of functions, No. 17; in russian; english translation in Jour. Sov. Math. 37 (1987), 802-822.

[13] S. Hawking AND G. Ellis, The large scale structure of space-time, Cambridge University Press, Cambridge, 1973.

[14] S. Klainerman and M. Machedon, Finite energy solutions of the Yang-Mills equations in $\mathbf{R}^{3+1}$, Ann. of Math. (2), 142 (1995), pp. 39-119.

[15] R. RACKE, Lectures on nonlinear evolution equations, Friedr. Vieweg \& Sohn, Braunschweig/Wiesbaden, 1992.

[16] H. SEIFERT, Smoothing and extending cosmic time functions, Gen. Rel. Grav., 8 (77), pp. 815831.

[17] M. TAYLoR, Partial Differential Equations, Springer, New York, Berlin, Heidelberg, 1996. 